\title{
Structural Analysis of Thermal Shields During a Quench of a Torus Magnet for the $12 \mathrm{GeV}$ Upgrade
}

\author{
O. Pastor, T. Willard, P. K. Ghoshal, D. Kashy, M. Wiseman, V. S. Kashikhin, G. Young, L. Elouadrhiri, and C. Rode
}

\begin{abstract}
A toroidal magnet system consisting of six superconducting coils is being built for the Jefferson Lab $12 \mathrm{GeV}$ accelerator upgrade project. This paper details the analysis of eddy current effects during a quench event on the aluminum thermal shield. The shield has been analyzed for mechanical stresses induced as a result of a coil quench as well as a fast discharge of the complete magnet system. The shield has been designed to reduce the eddy current effects and result in stresses within allowable limits.
\end{abstract}

Index Terms-Eddy current, Lorentz forces, protection circuit, quench, SSC cable, superconducting magnet, torus, 3D simulations.

\section{INTRODUCTION}

$\mathbf{T}$ $\mathrm{HE}$ challenges with the $12 \mathrm{GeV}$ upgrade is the size and complexity of the magnet system that needs to be accommodated as a part of the magnet system in HALL B at Jefferson Lab [1], [2]. As part of the design and engineering task in building the torus, it is important to establish analytically that a magnet of this size, as shown in Fig. 1, can survive quench dynamics. The quench scenario that is summarized here is a magnet fast discharge from $3770 \mathrm{~A}$ to $0 \mathrm{~A}$ in less than five seconds. All superconducting coils are driven normal by "quench back" as a dump resistor in the protection circuit, as shown in Fig. 2, removes 50\% of the energy.

This paper presents the evaluation of the stresses in the aluminum shield when exposed to the fast discharge (considered to be the worst case). The heat shield is designed to have stresses below yield during this fast discharge. Yielding of the shield has the potential to create a thermal short between the shield $(80 \mathrm{~K})$ and the coil case $(4 \mathrm{~K})$. During subsequent operation, the increased heat load on the coil case could increase the temperature of the coil and result in future quenches of the magnet.

This analysis uses ANSYS Maxwell [3] to calculate forces on the shield due to quench. These forces are then applied to a structural model in ANSYS Mechanical [4] to calculate stresses and deflections.

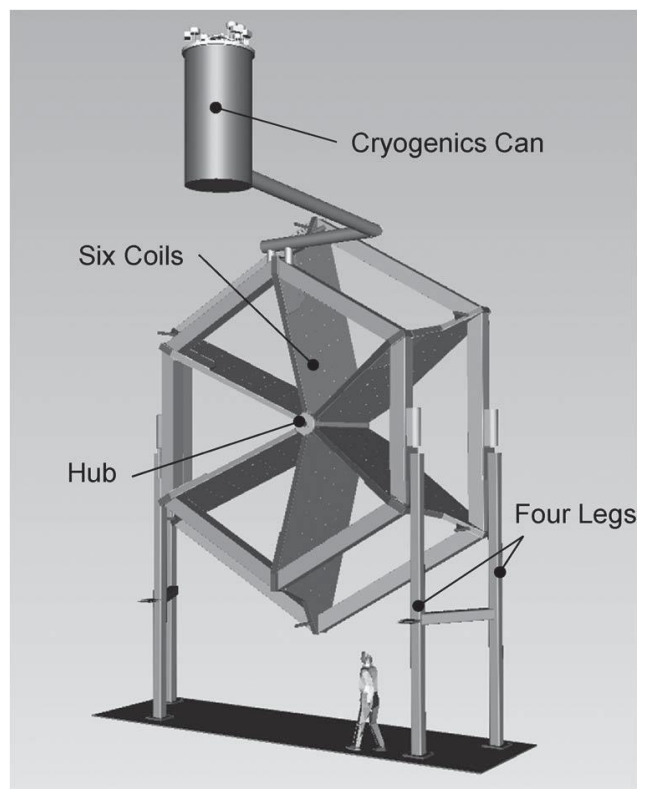

Fig. 1. CLAS12 Torus Magnet.

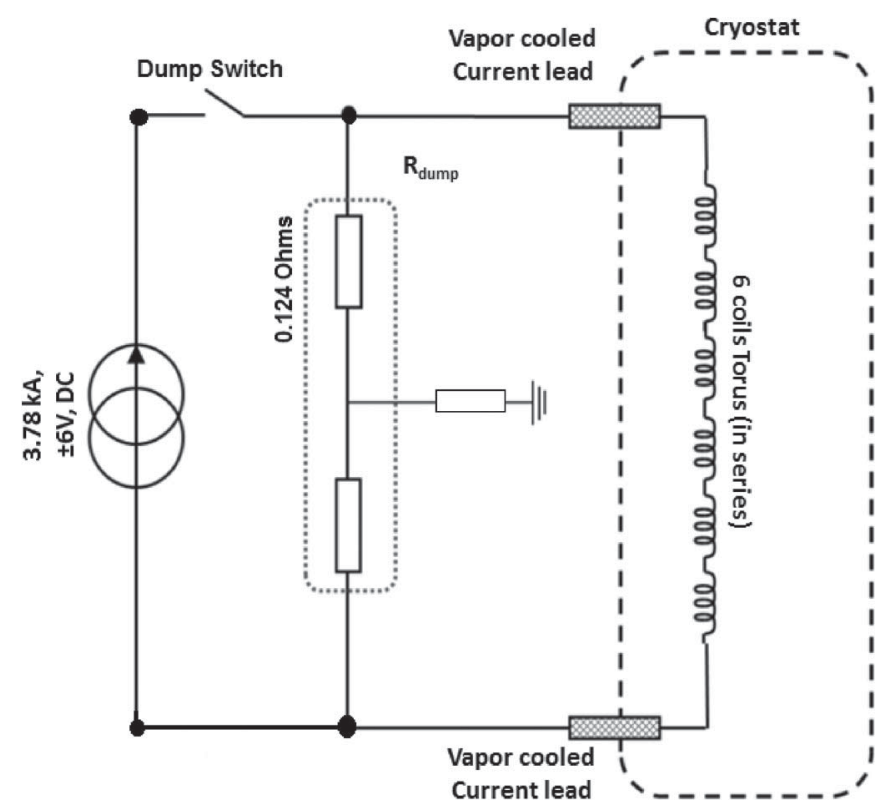

Fig. 2. Schematic arrangement of Torus magnet dump resistor in protection circuit.

\section{Magnet OvervieW}

Each of the six coils is vacuum-pressure impregnated with epoxy inside an aluminum coil case. The magnet coils are 

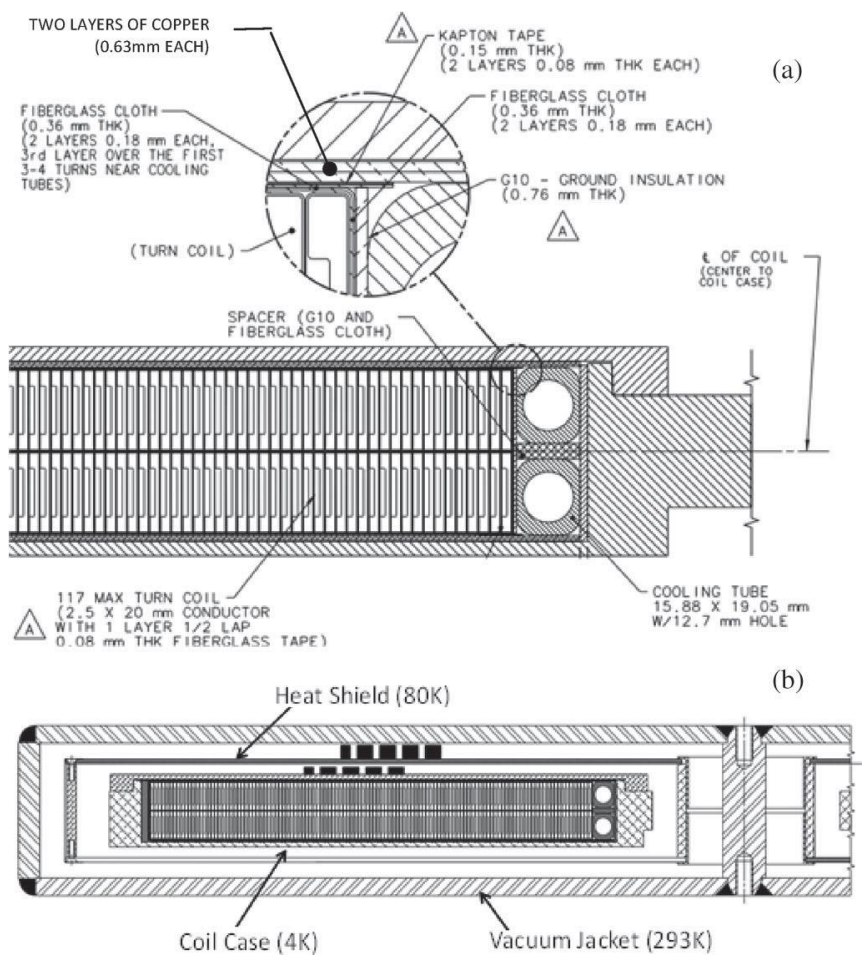

Fig. 3. (a) Construction detail for the torus coils, showing conduction cooling mechanism and coil winding details, (b) Cryostat Cross Section.

wound with Superconducting Super Collider, $36 \mathrm{NbTi}$ strand Rutherford cable soldered in a copper channel. On the outside of the coil are two layers of $0.635 \mathrm{~mm}$ thick copper. The copper sheets provide cooling from the $4.6 \mathrm{~K}$ (nominal) helium cooling tubes to the conductor and the coil case. The outer thickness of the coil case and cover is $3.6 \mathrm{~mm}$ thick aluminum. The $80 \mathrm{~K}$ thermal shield is $8 \mathrm{~mm}$ from the coil case surface. The stainless steel vacuum jacket is $12 \mathrm{~mm}$ from the heat shield. Cross sections of the coil and cryostat are shown in Fig. 3.

During a fast discharge, eddy currents are generated in the copper sheets, coil case and cover, and the thermal shield. These eddy currents result in forces normal to the surfaces that tend to push the affected areas inward toward the coil. The thermal shield was analyzed for this loading.

\section{Electromagnetic AnAlysis}

The magnet is analyzed operating at full current (3770 A) with a total magnet inductance $\left(\mathrm{L}_{\mathrm{T}}\right)=2.0 \mathrm{H}$ and storing 14.2 MJ. The dump resistor resistance of $0.124 \Omega$ is connected across the magnet transport current leads as the part of the magnet protection system. The event postulates all 6 coils (electrically connected in series) have quenched at the same instance. The normal resistance of each coil above the generation temperature, say at $10 \mathrm{~K}$, is $0.059 \Omega$ (This design evaluation uses a worst case resistance, 2.5 times more than copper resistance in our specification-providing enough margin that also includes the effect of magneto-resistance). The total resistance in the closed electrical loop circuit is about $0.478 \Omega$ (neglecting leads resistance $\sim 0.1 \mathrm{~m} \Omega$ ). The nor-

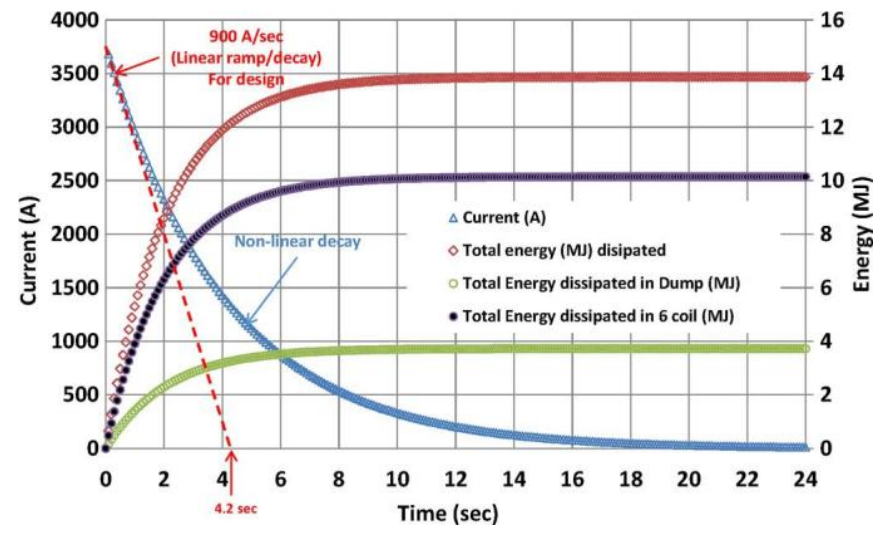

Fig. 4. Actual current ramp down vs. linear ramp down used for analysis and energy dissipated vs. time.

TABLE I

TORus CoIl PARAMETERS

\begin{tabular}{lcc}
\hline \hline \multicolumn{1}{c}{ Parameter } & Unit & Value \\
\hline Peak operating current & $\mathrm{A}$ & 3770 \\
Coil peak field & $\mathrm{T}$ & 3.58 \\
Number of coils & & 6 \\
Total number of turns/coil & $\mathrm{mm}$ & $2.5 \times 20$ \\
Superconducting cable dimensions & $\mathrm{mm}$ & 0.648 \\
NbTi strand bare diameter & & 36 \\
Number of strands in the cable & & 1.8 \\
Cu:Sc ratio (strand) & & 14.2 \\
Total stored energy & $\mathrm{MJ}$ & 2.0 \\
Inductance & $\mathrm{H}$ & \\
\hline \hline
\end{tabular}

mal time constant ( $T=$ time constant in seconds) is calculated as:

$$
T=\frac{L_{T}}{\left(6 \times R_{\text {coil }}+R_{\text {dump }}\right)}=\frac{2 H}{0.478 \Omega}=4.184 \mathrm{sec} .
$$

Looking at the exponential decay of the current shows that during the first $0.1 \mathrm{sec}$, the rate of change of current $(\mathrm{dI} / \mathrm{dt})$ is approximately $\sim 900 \mathrm{~A} / \mathrm{sec}$ (for this design evaluation, we considered the coil only and $\mathrm{dI} / \mathrm{dt}$ is primarily dominated by the superconducting coil and not the coil case). For the purpose of the structural analysis, a linear ramp down rate of $1000 \mathrm{~A} / \mathrm{sec}$ is assumed and analyzed. By using the higher linear ramp rate for the analysis, the design is conservative in terms of stress and deflection. A plot of the exponential decay and the linear rate used in the analysis is shown in Fig. 4. Not considered in this calculation is the mutual coupling between coil and shroud that retards $\mathrm{dI} / \mathrm{dt}$ and decreases ramp down rate by a factor of 2.5 [5].

The TORUS magnet electrical parameters as listed in Table I.

A transient analysis was performed using ANSYS Maxwell to calculate eddy current forces. The model included a single coil and thermal shield. Early analyses including the aluminum coil case showed that inclusion of the coil case reduced the 


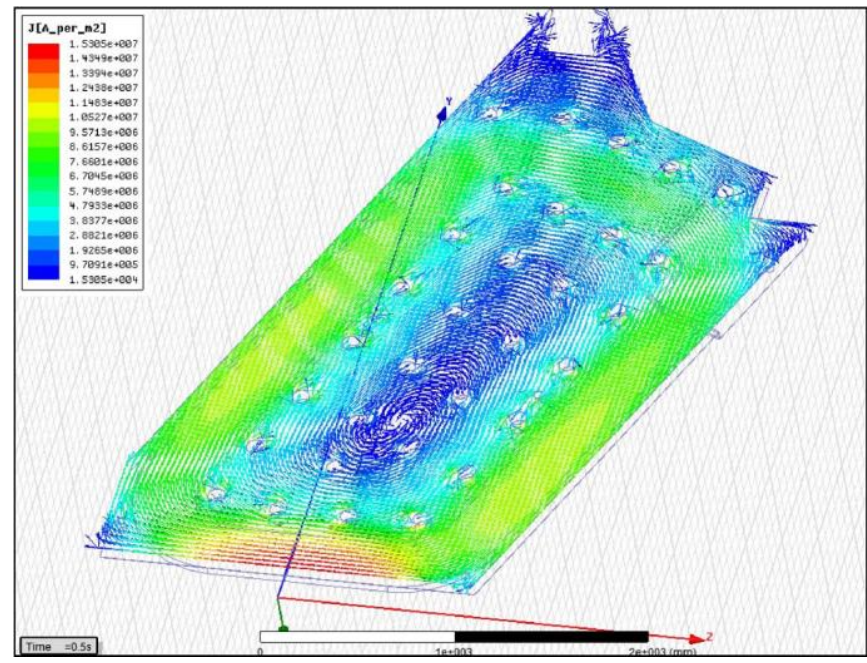

Fig. 5. Current density vector plot for solid thermal shield design.

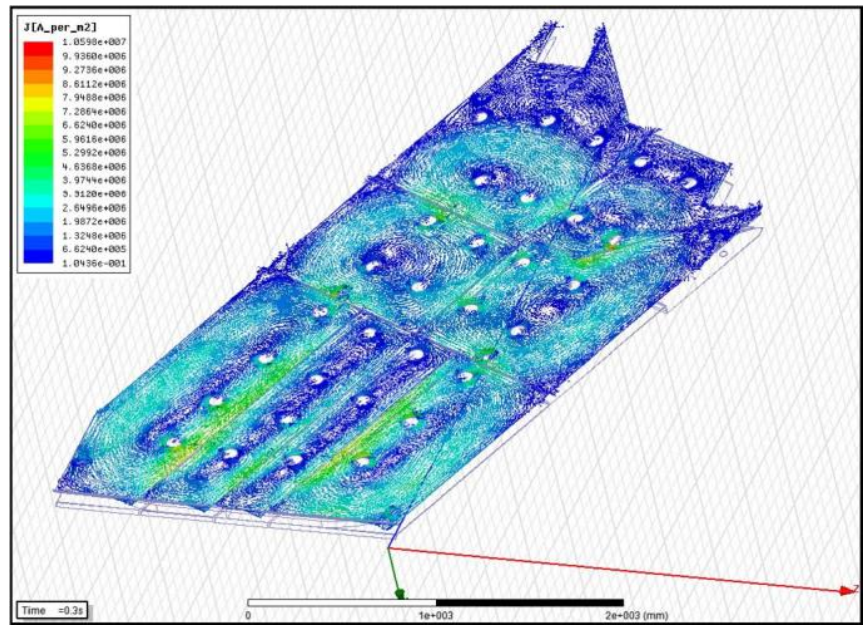

Fig. 6. Current density vector plot for segmented thermal shield design.

eddy currents in the shield. To reduce the model size, subsequent analyses used only the coil and shield. This adds some conservatism to the structural analysis. The heat shield model is 6061-T6 aluminum. The dimensions of the shield are approximately $4 \mathrm{~m} \times 2 \mathrm{~m} \times 3 \mathrm{~mm}$ thick. The heat shield has been designed as individual panels connected with G10 spacers. Using individual panels, compared to using one large plate, breaks up the eddy current paths thus reducing the out of plane forces on the shield. Current density vector plots for a continuous shield and the segmented shield are shown in Figs. 5 and 6. The slits, also known as eddy current breaks, were engineered using numerous simulations. The slit placement avoids any local concentration of eddy current loops that exist near the high magnetic flux density location at the hub where flux from the other five coils superposes on the coil we analyzed.

For the continuous shield, the majority of the current density is between $7 \times 10^{6} \mathrm{~A} / \mathrm{m}^{2}$ to $12 \times 10^{6} \mathrm{~A} / \mathrm{m}^{2}$ (average of $9.5 \times$ $10^{6} \mathrm{~A} / \mathrm{m}^{2}$ ) with local peaks about $15 \times 10^{6} \mathrm{~A} / \mathrm{m}^{2}$. For the segmented shield, the majority of the current density is about $2 \times 10^{6} \mathrm{~A} / \mathrm{m}^{2}$ to $3 \times 10^{6} \mathrm{~A} / \mathrm{m}^{2}$ (average of $2.5 \times 10^{6} \mathrm{~A} / \mathrm{m}^{2}$ ). The local peak spot of about $10 \times 10^{6} \mathrm{~A} / \mathrm{m}^{2}$ is attributed to the modeling anomalies and is neglected.

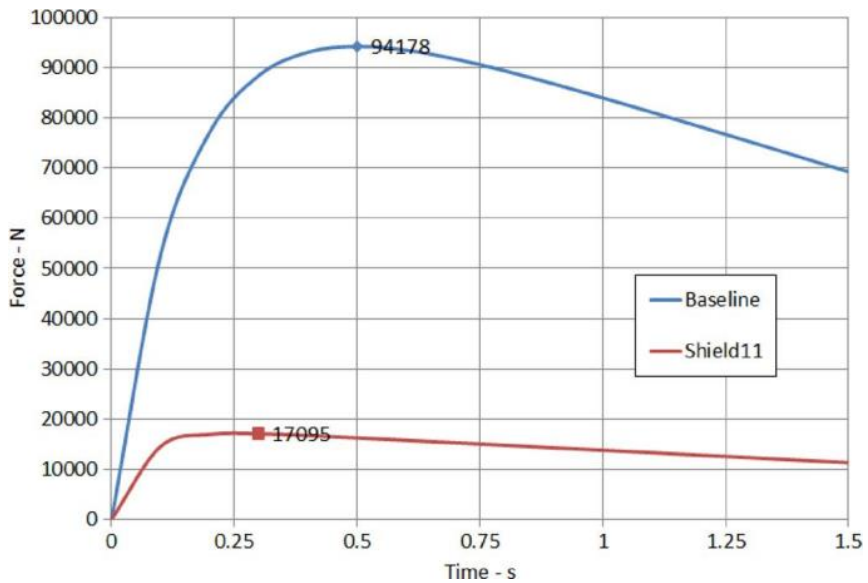

Fig. 7. Comparison of out of plane forces in a continuous shield and a segmented shield.

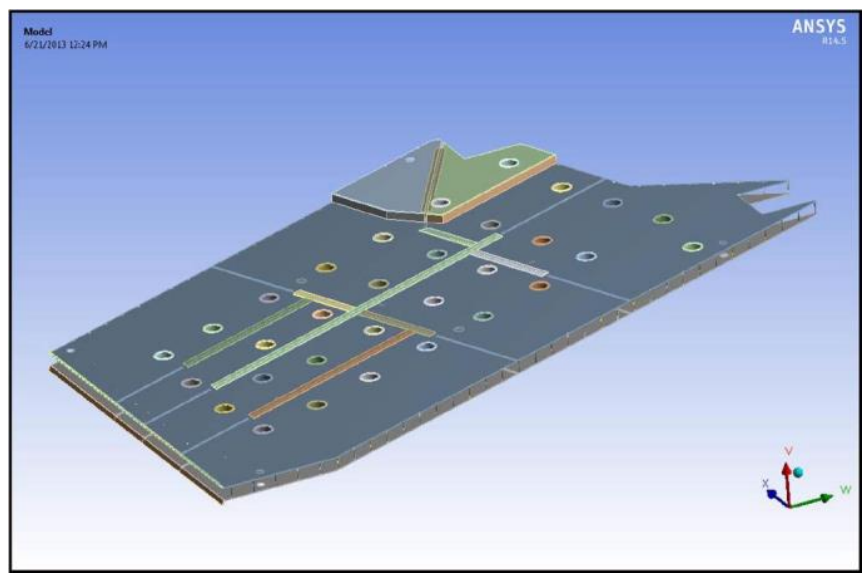

Fig. 8. Single thermal shield model of one coil.

The segmented shield reduces out of plane, Lorentz forces by $80 \%$ (from $94 \mathrm{kN}$ to $17 \mathrm{kN}$ ) compared to the continuous sheet due to the lower eddy currents. The maximum out of plane force occurs at 0.3 seconds after initiation of the fast dump. The loads at 0.3 seconds are used in a quasi-static analysis to assess the shield for stresses and deflections. The single coil quench case of the earlier analysis, carried out on the coil case and the superconducting coils, shows that quench-back is expected within $0.3 \mathrm{sec}[6]$. This interval suggests that the magnet with a linear discharge rate ramps the current down within $8.7 \mathrm{sec}$. This discharge rate is slower than the discharge rate $(3.8 \mathrm{sec})$ used for the analysis of the Torus shield, resulting in a conservative design. A comparison of the out of plane forces in a continuous shield and a segmented shield is shown in Fig. 7.

\section{Structural Analysis}

The forces were imported into a structural model and analyzed with ANSYS Mechanical. Since all coils are ramped down simultaneously, the eddy currents in each shield are the same. This is taken advantage of in the modeling of the shield geometry. A single shield is modeled for the eddy current analysis. The shield model is shown in Fig. 8.

The material strengths used to assess the stresses are the strengths at $80 \mathrm{~K}$. The shield is designed with a series of 


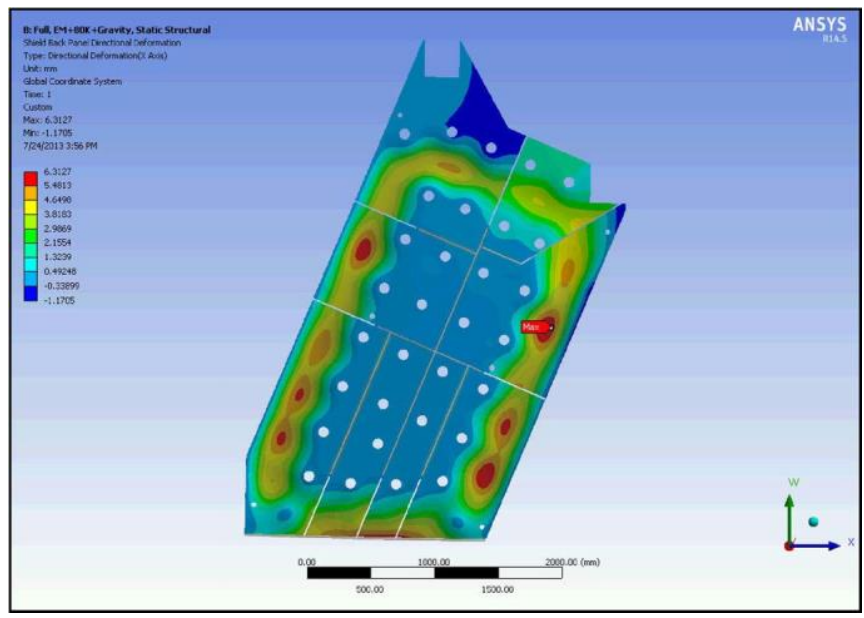

Fig. 9. Single shield deformations in the thermal shield.

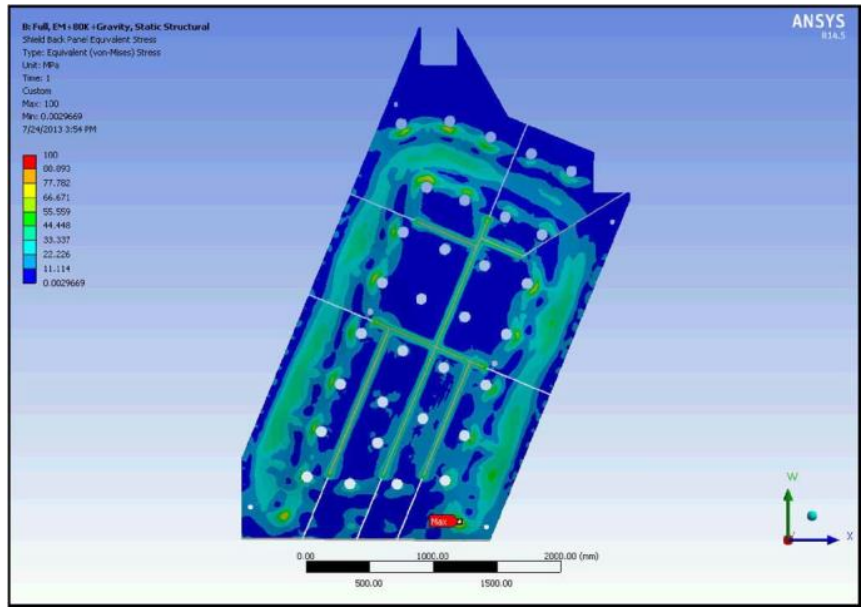

Fig. 10. Single shield stresses in the thermal shield.

bumpers that have two different lengths. The long bumpers are always in contact with the coil case and keep the shield from compressing the Multi-Layer Insulation (MLI) between the shield and the case. The short bumpers are not normally in contact with the coil case, and might only come into contact with the case during a fast discharge. The bumpers help to reduce the stresses and deflections of the shield. The deflections from the analysis for a ramp down rate of $1000 \mathrm{~A} / \mathrm{s}$ are shown in Fig. 9. The maximum deflection in the shield is $8 \mathrm{~mm}$. The stress results of the analysis on the shield are shown in Fig. 10. The maximum stress on the shield is $100 \mathrm{MPa}$, well within the yield strength (332 MPa) of the 6061-T6 AL at $80 \mathrm{~K}$ [6], [7].

In addition to the global stresses in the shield panels, the analysis looked at the side support pins that are located at the edges of the panels. A sub-model was used to look at the stresses in the pins and the sub-model stress results are shown in Fig. 11. The stresses in the heat shield and support pins are all below the yield strengths at $80 \mathrm{~K}$. In addition, the stresses in the G10 spacers were also assessed. Plots are not included here for the spacers, but the stress values are included in the factors of safety as listed in Table II.

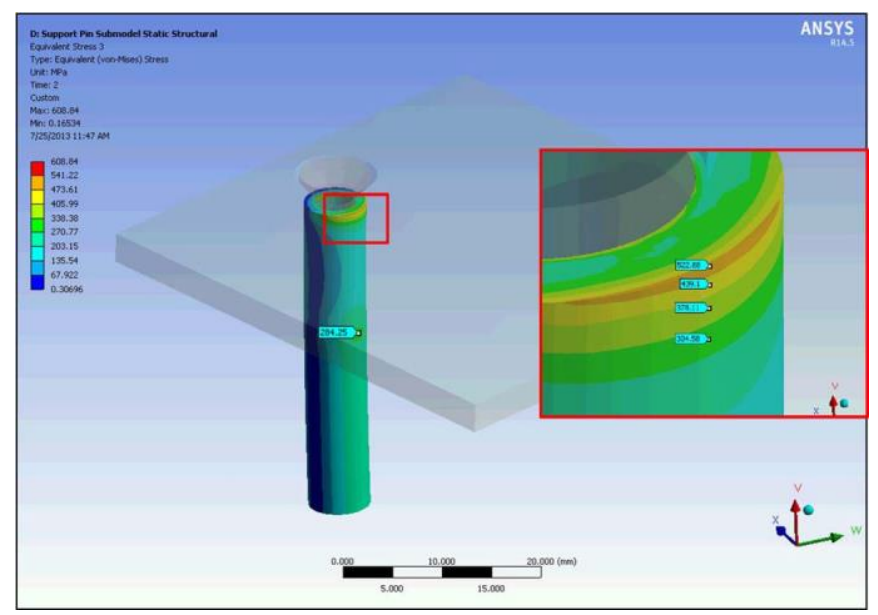

Fig. 11. Stresses in the support pin sub-model.

TABLE II

FACTORS OF SAFETY

\begin{tabular}{cccc}
\hline \hline Component & Stress & \multicolumn{2}{c}{ Factors of Safety } \\
& & Yield & Tensile \\
\hline $\begin{array}{c}\text { Aluminum } \\
\text { Shield }\end{array}$ & 106 & 3.13 & 3.98 \\
Edge Pins & 284 & 1.19 & 1.51 \\
G10 Spacers & 126 & - & 3.64 \\
\hline \hline
\end{tabular}

Since the analysis is conservative, a minimum factor of safety of 1.0 is used for the stress limits.

\section{CONCLUSion}

The segmentation of the shields reduces the current density from $9.5 \times 10^{6}$ to $2.5 \times 10^{6} \mathrm{~A} / \mathrm{m}^{2}$ with corresponding significant reduction of out of plane forces from $94 \mathrm{kN}$ to $17 \mathrm{kN}$.

The Torus magnet heat shield has been designed and analyzed for a fast discharge of $1000 \mathrm{~A} / \mathrm{s}$. This assumption is conservative not from stress or deflection, but from actual possible ramp rate. The analysis shows that the shield component stresses are below yield for this scenario.

\section{REFERENCES}

[1] C. H. Rode, "Jefferson Lab $12 \mathrm{GeV}$ CEBAF upgrade," in Proc. Adv. Cryogenic Eng., Trans. CEC, 2010, vol. 55, pp. 26-33, CP 1218.

[2] R. J. Fair and G. L. Young, "Superconducting magnets for the $12 \mathrm{GeV}$ upgrade at Jefferson laboratory," IEEE Trans. Appl. Supercond., to be published.

[3] ANSYS Maxwell, Release 15.0

[4] ANSYS Mechanical, Release 14.5.

[5] V. S. Kashikhin et al., "TORUS CLAS12-Superconducting magnet quench analysis," IEEE Trans. Appl. Supercond., vol. V24, no. 3, Jun. 2014, Art. ID. 4500405.

[6] H. Pan, S. O. Prestemon, S. Virostek, R. Preece, and M. A. Green, "Eddy-currents and the force analysis for the thermal shields of the MICE spectrometer solenoids," Oct. 2012, MICE Note 420. [Online]. Available: http://www.mice.iit.edu/micenotes/public/pdf/MICE0420/MICE0420

[7] J. E. Jenson et al., "Brookhaven National Laboratory selected cryogenic data notebook," Brookhaven National Laboratory Associated Universities Inc., Washington, DC, USA, 1980, U.S. DOE. 